\title{
Programmable Intrathecal Morphine Pump for the Management of Chronic Pain
}

\author{
Hyung Sug Oh, Hansan Oh, Kwan Ho Park ${ }^{\bowtie}$, Tae Wan Kim \\ Department of Neurosurgery, VHS Medical Center, Seoul, Korea
}

\begin{abstract}
There are a variety of options for the treatment of chronic pain. Oral medications include nonsteroidal anti-inflammatory drugs (NSAIDs), acetaminophen, antidepresssants, anticonvulsants and opioids. Common side effects of opioid administration are sedation, dizziness, nausea, vomiting, constipation, physical dependence, tolerance and respiratory depression. There are interventional techniques that involve injections into or around various levels of the spinal region. Intrathecal drug delivery systems (IDDS) for the treatment of pain is a modulator of nociceptive signal transmission to and within the central nervous system. IDDS consist of a pump and catheter, both of which are surgically placed under the skin. The implantable pump that stores and delivers medication through a catheter to the intrathecal (IT) space. IDDS has become one of the most innovating procedures in treating pain. Programming by external programmer over the implanted pump change the mode of drug delivery. They have the advantage of reduced oral or parenteral medication doses, side effects of long-term use, and a lower risk of infection. Clinician choose IDDS as a final method to relieve intractable pain. Morphine is the only opioid approved by FDA for IT administration and has been increasingly utilized for this purpose. Implantable IT morphine pump has become a common option for administering opiate medication for refractory chronic pain. Clinician should be aware of patient selection, surgical techniques, mechanical, pharmacological, and patient-specific complications.
\end{abstract}

Key Words: Intrathecal drug delivery systems; Chronic pain; Morphine pump.

ه Corresponding author: Kwan Ho Park, Department of Neurosurgery, VHS Medical Center, 53 Jinhwangdo-ro 61- gil, Gangdong-gu, Seoul 05368, Korea. Tel: 82-2-2225-1363, Fax: 82-2-2225-4152, E-mail: spineho@naver.com

\section{INTRODUCTION}

Analgesia can be administered by many different routes such as oral, intravenous, subcutaneous, transdermal, transmucosal, and intrathecal (IT). After many years of continuous oral analgesics, the daily dose can escalate to a point of intolerable side effects or ineffective pain control. However, in $10 \%$ to $30 \%$ of patients treated for chronic pain, they fail to obtain adequate analgesia with oral analgesics ${ }^{6}$.

IDDS are highly complex systems. The IDDS is composed of a pump that stores the drug in a refillable reservoir and delivers it to the IT space via a catheter (Fig. 1A, B). A replaceable battery powers the drug delivery, and an external device programs drug delivery parameters. IT therapy should be considered after conservative treatments have failed or patients have significant side effects with systemic analgesics. The American Society of Interventional Pain Physicians recommends IT analgesia route "after all other methods have failed including conservative and surgical treatment ${ }^{25}$. In 1991, externally programmable, batterypowered IDDS pumps were introduced, allowing for noninva- sive dose changes of these medication.

There are no universally accepted guidelines or recommendations for patient selection. Indications for IDDS therapy include post-spinal surgery syndrome (PSSS), diabetic peripheral neuropathy, complex regional pain syndrome (CRPS), and spasticity. Patients with these conditions can benefit the most from IDDS therapy ${ }^{8,18}$. Patients with chronic noncancer pain being considered for IDDS must evaluate psychiatric comorbidities such as depression, anxiety, addiction, suicidal ideation, or personality disorders. These diseases have been associated with a poor response to IT therapy ${ }^{3}$.

The contraindications for IDDS include systemic infection, uncontrolled coagulopathy allergy to morphine, high cerebrospinal fluid (CSF) pressure, a failed trial of spinal opioid therapy, spinal deformity, and psychiatric and behavioral comorbidities $^{25,31}$. Under meticulous approach, good surgical techniques and patient adjustments of intrathecal dosage, programmable intrathecal morphine pump may provide a alternative therapy with lower doses and an fewer side effects. 


\section{INTRATHECAL MEDICAITIONS}

Morphine is the only opioid approved by the Food and Drug Administration (FDA) for the IT route. Implanted programmable pump for the delivery of IT morphine delivers morphine directly into the CSF space, which provides better analgesia at lower dosage and minimal adverse effects ${ }^{25,31}$. Low dose medications can provide profound pain relief because many analgesia receptors are located in the spinal cord. IT opioids act at the substantia gelatinosa in the dorsal horn of the spinal cord at specific $\mu, \kappa$, and $\delta$ receptors. The pharmacology of morphine is based on its predominant interaction with the $\mu$ receptor. Morphine binds to receptors on the primary afferent neurons (presynaptic) and cells within the dorsal horn of the spinal cord (postsynaptic) to inhibit the release of neurotransmitters like substance $\mathrm{P}$ and calcitonin gene-related peptide and hyperpolarize postsynaptic neurons, respectively. Morphine remains the gold standard of IT therapy.

To reduce risk for morbidity and mortality in IDDS, the Polyanalgesic Consensus Conferende (PACC) group outlined their most recent recommendations on drug choice, concentration limits, and recommended starting dosages in their 2012 guidelines". Programmable morphine pump is a continuous, controlled and reversible drug delivery system in which the drug dosage can be adjusted as per the requirement for pain relief and refilled without any major procedure. Clinician can easily interrogate this information with the programmer noninvasively ${ }^{25,31)}$. To provide the same amount of pain relief, IT morphine requires a much lower dosage than oral, intramuscular, intravenous or epidural injections and, consequently, has much less systemic side effects ${ }^{19,25)}$. IT morphine is approximately 10 times more potent than the same dose administrated epidurally. Its response can be evaluated by a screening test prior to system implantation. Pump should only be implanted if the patient has experinced successful pain control $(>50 \%)$ with a screening test injection of spinal epidural $(5-10 \mathrm{mg})$ or intrathecal $(0.5-2 \mathrm{mg})$ morphine after stopping all analgesics and the life expectancy of the patient should be more than 3 months ${ }^{19,25)}$.

Sincee chronic infusions of morphine do yield potent metabolites, the role of metabolites is important. Morphine-3-glucuronide (M3G) plays a large role with chronic infusions. The potency of IT M3G is approximately 10 to 45 times that of morphine and associated with sedation, hyperalgesia, and myoclonus ${ }^{2)}$. Morphine-6-glucuronide (M6G) is associated with chronic nausea and vomiting and profound sedation, leading to respiratory depression. High concentration of metabolites can cause cognitive deficits, impaired attention and memory ${ }^{27,30)}$.

Despite the increased complexity, there are many benefits of IT over oral or parenteral routes. IT morphine injection greatly reduce morphine metabolites ${ }^{5}$. An additional benefit is that the IT route delivers morphine in close proximity to the spinal cord $^{7,14)}$. Patients experience fewer side effects associated with opioid overdose.

\section{TRIALING}

It is commonly recommended to perform a trial prior to implantation because it is currently the most demonstrative method of emulating a system that would eventually be implanted. The British Pain Society and Polyanalgesic Consensus Conference (PACC) recommends that a trial should be performed before implant ${ }^{11,32)}$. After determining that a patient is a candidate for IT pump implantation, the trialing based upon the pain type was performed. Trials are consisted of either bolus or continuous dose delivered by an epidural or IT route. Trial options include single bolus, multiple single boluses over several times, or administrated continuously via catheter. If the patient cannot tolerate a continuous infusion trial, then one may administer a bolus injection trial of morphine epidurally or intrathecally. The bolus trial is requiring shorter hospital observation than continuous mode. A single bolus trial for chronic pain, the risks of adverse events are potentially greater. There may be a greater potential for a placebo effect during a single bolus trial due to patients' self-reports of pain. Continuous IT trials may mimic the results of IDDS. The continuous trial increases the risk for infection and spinal cord injury. continous IT trialing via an indwelling catheter may offer the opportunity to assess an efficacy versus side effects of implanted pump. During IT or epidural catheter trial, the catheter tip should be placed fluoroscopically at the level where permanent catheter is planned. The typical IT morphine bolus dose was $1 \mathrm{mg}^{1,24)}$. Others have based the dose on calculated morphine equivalent pretrial dose and gave $1 / 100$ or $1 / 200$ of that dose for the IT bolus trial ${ }^{28)}$. Continuous morphine doses ranged from 0.025 to $11.28 \mathrm{mg} /$ day $^{20)}$. For continuous IT catheter trial, it was suggested that morphine sulfate be initiated at $0.2 \mathrm{mg} /$ day with titration to approximately $2 \mathrm{mg} / \mathrm{day}^{23}$. Others used $1 / 300$ of the calculated morphine equivalent dose as the continuous trial $\operatorname{dos}^{22)}$. No one method has been shown to be better than the other ${ }^{10-13)}$.

Trial accurately predicts the efficacy, success, and adverse events after implanting. The most common goals are pain relief, reduction of systemic opioid use, and functional improvement ${ }^{11}$. A $50 \%$ pain relief as well as a favorable side effect profile are considered as predictive of a successful IDDS implantation ${ }^{21}$. The PACC guidelines strongly recommend at least 24-hour inpatient observation for trialing ${ }^{11,32)}$. A wide variety of non-nociceptive side effects may also occur in susceptible patients. The side effects are mostly mediated by opioid receptors. Treatment usually involves the utilization of naloxone. The expert consensus guidelines of PACC utilize critical evaluation of existing data and clinical panel discussions to formulate recommendations for clinicians. The 2012 PACC guidelines provided a summary of the pharmacology of IT analgesics. It also included additional sections on trialing methods, morbidity and mortality of IT drugs, and catheter granulomas ${ }^{9}$.

Pruritis, nausea, vomiting, and urinary retention are the most common side effects of IT morphine. Sedation and respiratory 
depression are one of the serious side effects. Patients considering IT morphine pump therapy should be informed and advised about the possible side effects associated with long-term IT morphine administration prior to placement of a permanent morphine infusion pump.

\section{TYPES OF PUMPS}

The first commercially available implantable programmable pump was the Medtronic SynchroMed ${ }^{\circledR}$ pump originally released for cancer chemotherapy in 1988. The most commonly implanted pump is the Medtronic SynchroMed ${ }^{\circ}$ II infusion pump that can be noninvasively programmed. The pump reservoir is called a metal bellows reservoir that contains the drug. The surrounding pressurized gas exerts pressure on the bellow, which pushes the drug from a reservoir through a valve and into a catheter. This pressurized gas system will deliver a different drug amount according the changes in atmospheric pressure and body temperature. Therefore, the patient is advised to avoid these conditions.

Programmable pumps allow the clinician to vary infusion rates to increase or decrease the dose without changing the concentration of the drug or drugs in the pump. It is useful for patient who has conditions that requires alternations of doses. Medtronic SynchroMed ${ }^{\mathbb{E}}$ II allow patient-controlled intrathecal bolus dosing via remote control device. It uses a peristaltic roller system to move the drug from the reservoir to the implanted intrathecal catheter. By using an external device, personal therapy manager $\left(\right.$ myPTM $\left.^{\circledR}\right)$ (Fig. 1C), clinician can activate the SynchroMed $^{\circledR}$ infusion system to give a bolus in addition to the continuous infusion. It was programmed for clinician to control the bolus dose, the lockout time, and the total number of doses a patient can receive per day. This allows patient to customize their pain control to cover variations in chronic pain. In cases of suspected malfunction, the pump can be interrogated or deactivated without emptying the drug reservoir. The negative pressure draws medications from the syringe during pump refills rather than requiring positive pressure from the syringe plunger. The pump battery has a 4-7 year life span before requiring surgical replacement. In 2012, two new programmable pumps were approved by the US Food and Drug Administration : Prometra $^{\circledR}$ and MedStream ${ }^{\text {TM }}$.

\section{SURGICAL TECHNIQUES}

\section{Sterile technique}

Good sterile technique and pre-operative antibiotics are the most important controllable factors in preventing wound infections. Skin preparation with chlorhexidine-alcohol solutions has been shown to be superior to povidone-iodine solutions. Careful cleansing of both the surgeon's hands and the incision site reduces skin bacterial counts. Prophylactic systemic antibiotics against the most likely pathogens should be administrated intravenously and within 30 minutes of skin incision. Surgeon mostly focused on the surgical field but should also pay attention to the instrument table and specifically the handling of the pump. Sterility of the C-arm drape and duration of hardware exposure in the operating room are also important.

\section{Catheter placement and anchoring}

Prior to needle insertion, the spinous processes of the levels of insertion should be marked on the skin. Insert the introducer needle using shallowing angle, paramedian fashion. An angle of approximately 30 to 45 degrees off of the spine is ideal. The skin entry point will be 1 to $2 \mathrm{~cm}$ lateral to the midline on the side of the intended pump pocket, and 1 to 1.5 vertebral levels below the intended interlaminar space for dural puncture. The typical entry site will range from the L2 to L4 interlaminar spaces. With the stylet in place, the introducer needle should be advanced to the superior edge of the caudad lamina for the target space. A needle advanced into the IT space. The fluoroscopic view should be the lateral view to monitor the needle depth in the IT space. With gentle aspiration, the needle is advanced until good CSF flow. Replace the needle stylet to stop CSF flow
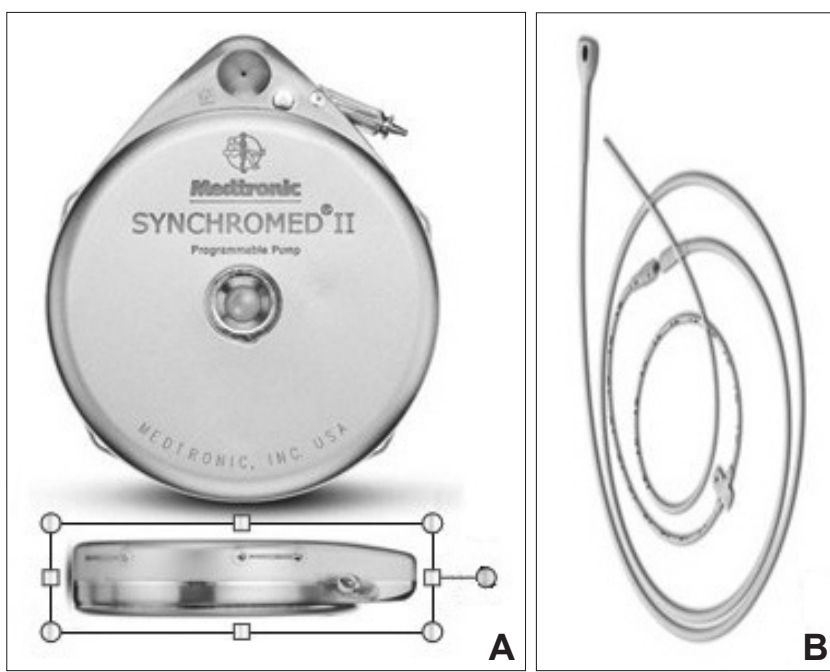

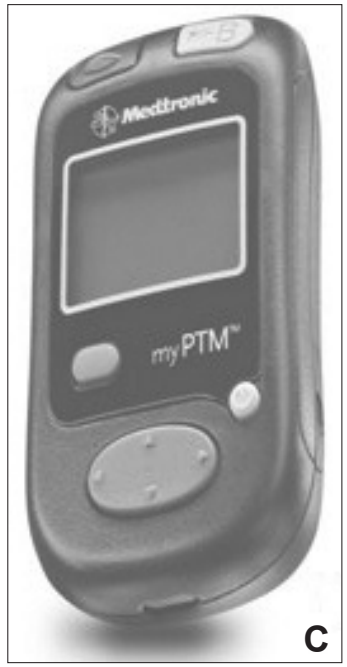

Fig. 1. The implantable intrathecal pump system. A: Programmable pump. B: Intrathecal catheter. C: Personal therapy manager $\left(\right.$ myPTM $\left.^{\circledR}\right)$. 
until ready to place the catheter. The catheter guide wire is fully advanced into the catheter and is in place during insertion and positioning of the catheter. Under fluoroscopic guidance, the tip of the catheter is at desired location. Leaving the needle in place to protect the catheter, make a $5 \mathrm{~cm}$ vertical incision in the midline over the spinous processes down to the dorsolumbar fascia, exposing the adjacent supraspinous ligament. The edges of the incision should be undermined to expose the fascial plane for the anchoring hardware to rest in and to permit gentle bending of the catheter. With the catheter guide wire still in place, carefully remove the needle and then withdraw the guide wire. The CSF flow should be confirmed after the needle and stylet are removed and the suture is securely completed. Bring the external portion of catheter through the skin into the pocket. The end of the catheter is clamped to prevent CSF loss while anchoring and tunneling. After placing a suture through the supraspinous ligament, slide butterfly anchor over the catheter down to the fascia. Then tie the two wings of the butterfly anchor together with the suture. A second suture placed through the supraspinous ligament and tied around the neck of butterfly wings. Good CSF flow from the catheter is reconfirmed and the catheter's end is clamped to prevent further CSF loss.

\section{Pump pocket}

The successful IDD implant is to plan the location and orientation of the pump, pocket, and incision with the patient. One direct attention to the lower quadrant of the abdomen and the desired pocket for the pump. A majority of pumps are placed in the lower quadrant of the abdomen. The pump should not con- tact the iliac crest, pubis symphysis, or lower costal margins ${ }^{4,299}$. The pump should not contact the iliac crest, pubis symphysis, or lower costal margins. The clearance of these bony borders should be checked when the patient is sitting, standing, and lying supine. These measurements minimize discomfort and possible pump damage. An alternative location is the posterior flank4.

A $10-12 \mathrm{~cm}$ transverse incision is made down to the subcutaneous layer beneath the superficial fatty plane of Camper's fascia and the membranous plane of Scarpa's fascia. The base of the pocket will usually be the fascia of the external oblique or the rectus abdominis. Hemostasis with electrocautery is necessary. Incision extended to a depth of a least $1.5 \mathrm{~cm}$ but no more than $2.5 \mathrm{~cm}$. A oversized pocket increases the risk of seroma and shifting of the pump. The pocket should be undermined predominantly cephald or cauadal to the incision. After ensuring proper fit of the pump, anchoring sutures must be placed with in the base of the pocket at least three corners. These sutures should be firmly anchored to the fascia if possible.

\section{Tunneling}

Once the pocket is prepared, the catheter must be tunneled from the posterior incision to the pump pocket. The provided tunneling device may be bent in a gentle curve to facilitate passage subcutaneously from posterior to anterior trunk. The catheter is tunneled into the pump pocket using tunneling device. Unclamp the catheter tip and eusure patency by observing CSF flow from the catheter. Attach the catheter to the provided connector. The connector is then attached to the pump. Insert the pump into its pocket and suture to tie it down in three of the cor-
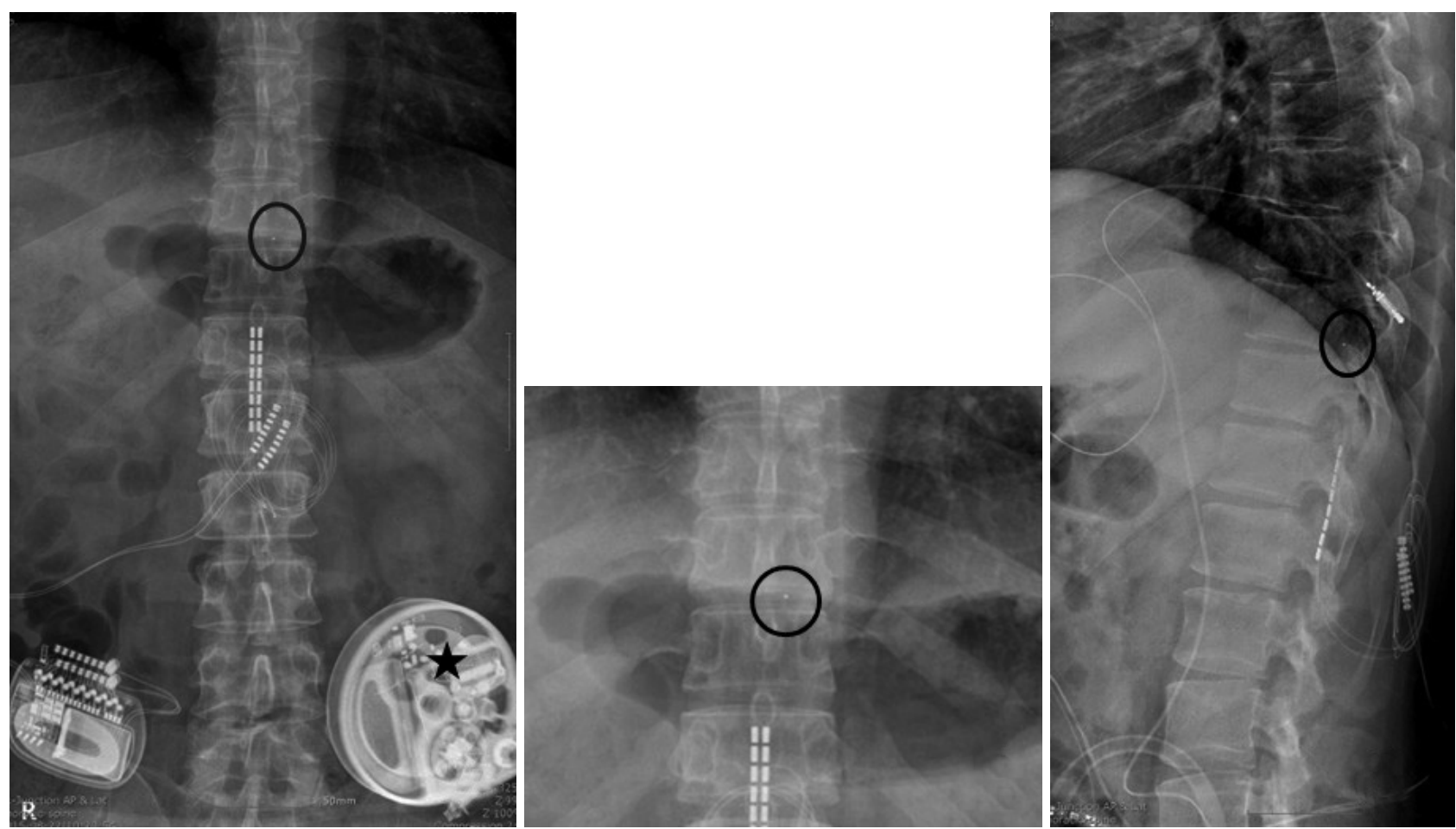

Fig. 2. The intrathecal pump system consists of a pump and a catheter that carries morphine from the pump to the spinal cord and nerves. Post-implantation radiographs show a pump $(\star)$ and a catheter tip placement (black circle). 
ner suture loops. Place coils of excessive catheter behind the pump and ensure that the pump refill port is facing up.

\section{Closure}

Before closure of the posterior pocket, one suture placed through the thoracolumbar fascia on one side of the anchor then crossed over to the other side to close a flap tissue over the top of the anchor. This tight pocket over the anchor will help preventing catheter migration out of the IT space. For the pump site, only the subcutaneous fascia will require closure. Skin closure of both incisions are performed. The pump is programmed to the desired continous setting (Fig. 2).

\section{COMPLICATIONS}

The complications from the implantation of an intrathecal pump can becategorized as surgical, pump, or catheter related, and the frequency was shown to be $10 \%, 35 \%$, and $65 \%$, respectively $^{33)}$. The surgical complications include bleeding, infection, CSF leaks, and neurological injury. Pump-related complications included pump repositioning, infection, and battery exhaustion. Catheter-related complications included catheter dislocation, disconnection, leakage, occlusion, and granuloma formation at the catheter tip ${ }^{15}$.

\section{Bleeding}

Intraoperative bleeding is most likely to be problematic in patients with a history of bleeding diathesis or hepatic disease, or those on anticoagulant therapy. It can occur from ineffective local hemostasis during the procedure. Although epidural or intrathecal bleeding is extremely rare, it is a much more serious morbidity. Postoperatively, it will present with increased back pain that rapidly progresses to neurological deficits. Later signs include fever and nuchal rigidity.

\section{Infection}

The use of strict sterile techniques, antibiotics, and monitoring is important to prevent infection. The most common complication is SSI ranging from $2.4 \%$ to $7 \%$. The incidence of infection following intrathecal pump implantation has been reported to be between $2.5 \%$ and $9 \%{ }^{16}$. The majority of infections occur at the pump pocket site and present within 15 to 45 days after implantation 18.

The clinical presentation of a local pump pocket infection are increased pain, tenderness, fever, erythema, swelling, drainage, and warmth at the surgical site. Patient with CSF infection and meningitis may present with headache, nuchal rigidity, nausea, vomiting, and fever. These patients may have an leukocytosis, C-reavtive protein (CRP), or erythrocyte sedimentation rate (ESR). In more than half of the infections, the primary organism was Staphylococcus aureus. Wound infections need to be treated early and aggressively to prevent serious complications. Superficial infections should be tested for the bacterial cultures and treated with appropriate antibitic therapy. In more serious infections such as epidural or intrathecal infections, the pump and catheter should be removed immediately, followed by appropriate antibiotic treatment. The diagnosis of intrathecal infection is conformed by positive bacterial CSF cultures.

\section{Cerebrospinal fluid leaks}

CSF leaks may occur in as much as $20 \%$ around the catheter via dural puncture site or as a result of catheter disconnection or fracture ${ }^{23)}$. The persistent leakage of CSF may result in a postdural puncture headache. In most instances these headaches will disappear over time. CSF leaks that are not catheter-related complications may be treated conservatively. Some develop nausea, vomiting, and severe headache.

\section{Catheter complications}

Damage to the nerve roots or the spinal cord itself during catheter insertion ccould occur, resulting in pain, sensory loss, bowel and bladder dysfunction, and/or weakness. The rate of catheter-related complications was $18.6 \%{ }^{17)}$. These complications include disconnection, fracture, puncture, and migration from the intrathecal space (Table 1). Catheter-related problems are the most common causes of failure following implantation of IDDS. Catheter migration is usually the result of improper anchoring of the catheter to the fascia. Catheter failure may present as inadequate analgesia, CSF leaks, or withdrawal symptoms. If CSF can be aspirated freely, fluoroscopic evaluation with contrast injection may help identify leaks and the location of catheter with in the intrathecal space. A computed tomography (CT) myelogram following injection of contrast through the side port may be required to identify the location of a leak or formation of a granuloma at the catheter tip.

\section{Granuloma formation}

The inflammatory response leads to the development of a granuloma. Granulomas are aseptic inflammatory masses composed of macrophages, neutrophils, and monocytes with granulation tissue. The formation of a granuloma at the catheter tip may cause a loss of analgesic efficacy, pain, and progressive neurologic symptoms despite escalating doses of IT morphine. Risk factors for granuloma formation include an increasing dosage and high concentration infusion of morphine. Imaging by magnetic resonance imaging (MRI) with contrast enhancement or CT myelogram can aid in the evaluation of a suspected granuloma. It is recommended that the concentrations of the medications be kept as low as possible to decrease the incidence of granuloma formation. Treatment options include replacement of the IT infusate with preservative free saline, removal of the catheter, or surgical exploration and mass resection.

\section{CONCLUSION}

IDDS with morphine progammable pump provides a treat- 
ment option when more conservative therapies have failed for chronic pain. The potential benefits to IT pump include the ability to deliver opioids where they work, increasing potency, and decreasing the systemic doses, thus reducing side effects. Good sterile technique during the surgical implantation as well as catheter anchoring can minimize the IDDS related complications.

\section{REFERENCES}

1. Anderson VC, Burchiel KJ, Cooke B : A prospective, randomized trial of intrathecal injection vs. epidural infusion in the selection of patients for continuous intrathecal opioid therapy. Neuromodulation $6: 142-152$, 2003

2. Bennett G, Serafini M, Burchiel K, Buchser E, Classen A, Deer T, et al. : Evidence-based review of the literature on intrathecal delivery of pain medication. J Pain Symptom Manage 20 : S12-S36, 2000

3. Brown J, Klapow J, Doleys D, Lowery D, Tutak U : Disease specific and generic health outcomes : a model for the evaluation of long-term intrathecal opioid therapy in noncancer low back pain patients. Clin J Pain 15 : 122 131, 1999

4. Buvanendran A, Jaycox M, Deer TR : Techniques of implant placement for intrathecal pumps in Deer TR (ed) : Intrathecal drug delivery for pain and spasticity, vol. 2. Philadelphia : Saunders, 2012, pp78-83

5. Chauvin M, Samii K, Schermann JM, Sandouk P, Bourdon R, Viars P : Plasma pharmacokinetics of morphine after i.m., extradural and intrathecal administration. Br J Anaesth 54 : 843-847, 1982

6. Cherny N, Ripamonti C, Pereira J, Davis C, Fallon M, McQuay H, et al. : Expert Working Group of the European Association of Palliative Care Network. Strategies to manage the adverse effects of oral morphine : an evidence-based report. J Clin Oncol 19 : 2542-2554, 2001

7. Cousins MJ, Mather LE : Intrathecal and epidural administration of opioids. Anesthesiology 61 : 276-310, 1984

8. Deer T, Winkelmuller W, Erdine S, Bedder M, Burchiel K: Intrathecal therapy for cancer and nonmalignant pain : patient selection and patient management. Neuromodulation 2 : 55-66, 1999

9. Deer TR : Polyanalgesic Consensus Conference--2012. Neuromodulation $15: 418-419,2012$

10. Deer TR, Levy R, Prager J, Buchser E, Burton A, Caraway D, et al. : Polyanalgesic Consensus Conference--2012 : recommendations to reduce morbidity and mortality in intrathecal drug delivery in the treatment of chronic pain. Neuromodulation 15 : 467-482; discussion 482, 2012

11. Deer TR, Prager J, Levy R, Burton A, Buchser E, Caraway D, et al. : Polyanalgesic Consensus Conference--2012 : recommendations on trialing for intrathecal (intraspinal) drug delivery : report of an interdisciplinary expert panel. Neuromodulation 15 : 420-435; discussion 435, 2012

12. Deer TR, Prager J, Levy R, Deer TR, Levy R, Prager J, et al. : Polyanalgesic Consensus Conference--2012 : recommendations for the management of pain by intrathecal (intraspinal) drug delivery : report of an interdisciplinary expert panel. Neuromodulation 15 : 436-464; discussion 464-466, 2012.

13. Deer TR, Prager J, Levy R, Rathmell J, Buchser E, Burton A, et al. : Polyanalgesic Consensus Conference--2012 : consensus on diagnosis, detection, and treatment of catheter-tip granulomas (inflammatory masses). Neuromodulation 15 : 483-495; discussion 496, 2012

14. Dickenson AH : Spinal cord pharmacology of pain. Br J Anaesth 75 : 193-200, 1995

15. Fluckiger B, Knecht H, Grossmann S, Felleiter P : Device-related complications of long-term intrathecal drug therapy via implanted pumps. Spinal Cord 46 : 639-643, 2008
16. Follett KA, Boortz-Marx RL, Drake JM, DuPen S, Schneider SJ, Turner MS, et al. : Prevention and management of intrathecal drug delivery and spinal cord stimulation system infections. Anesthesiology 100 : 1582-1594, 2004

17. Follett KA, Naumann CP : A prospective study of catheter-related complications of intrathecal drug delivery systems. J Pain Symptom Manage 19 : 209-215, 2000

18. Ghafoor VL, Epshteyn M, Carlson GH, Terhaar DM, Charry O, Phelps PK : Intrathecal drug therapy for long-term pain management. Am J Health Syst Pharm 64 : 2447-2461, 2007

19. Greenberg MS : Handbook of neurosurgery : Pain. ed 6. New York : Thieme Medical Publishers, 2006, pp376-400

20. Grider JS, Harned ME, Etscheidt MA : Patient selection and outcomes using a low-dose intrathecal opioid trialing method for chronic nonmalignant pain. Pain Physician 14 : 343-351, 2011

21. Hassenbusch SJ, Stanton-Hicks M, Covington EC, Walsh JG, Guthrey DS : Long-term intraspinal infusions of opioids in the treatment of neuropathic pain. J Pain Symptom Manage 10: 527-543, 1995

22. Kim D, Saidov A, Mandhare V, Shuster A : Role of pretrial systemic opioid requirements, intrathecal trial dose, and non-psychological factors as predictors of outcome for intrathecal pump therapy : one clinician's experience with lumbar postlaminectomy pain. Neuromodulation 14 : 165-175; discussion 175, 2011

23. Knight KH, Brand FM, Mchaourab AS, Veneziano G : Implantable intrathecal pumps for chronic pain : highlights and updates. Croat Med J 48 : 22-34, 2007

24. Kumar K, Kelly M, Pirlot T : Continuous intrathecal morphine treatment for chronic pain of nonmalignant etiology : long-term benefits and efficacy. Surg Neurol 55 : 79-86; discussion 86-88, 2001

25. Lee KS, Chu KS, Chung CL, Lin CL, Hwang SL, Howng SL : Intrathecaldrug delivery system with programmable morphine pump for pain of terminally Ill cancer patients. J Chinese Oncol 25 : 159-66, 2009

26. Manchikanti L, Abdi S, Atluri S, Benyamin RM, Boswell MV, Buenaventura RM, et al. : An update of comprehensive evidence-based guidelines for interventional techniques in chronic spinal pain. Part II : guidance and recommendations. Pain Physician 16 (2 Suppl) : S49-S283, 2013

27. McCracken LM, Iverson GL : Predicting complaints of impaired cognitive functioning in patients with chronic pain. J Pain Symptom Manage 21 : 392-396, 2001

28. Rauck RL, Cherry D, Boyer MF, Kosek P, Dunn J, Alo K : Long-term intrathecal opioid therapy with a patient-activated, implanted delivery system for the treatment of refractory cancer pain. J Pain 4 : 441-447, 2003

29. Rosenow JM : Surgical techniques for intrathecal medication delivery system implantation in Krames ES, Peckham PH, Rezai AR (eds), Neuromodulation. London : Elsevier, 2009, pp1027-1032

30. Sjogren $\mathrm{P}$, Thomsen AB, Olsen AK : Impaired neuropsychological performance in chronic nonmalignant pain patients receiving long-term oral opioid therapy. J Pain Symptom Manage 19 : 100-108, 2000

31. Stearns L, Boortz-Marx R, Du Pen S, Friehs G, Gordon M, Halyard M, et al. : Intrathecal drug delivery for the management of cancer pain : a multidisciplinary consensus of best clinical practices. J Support Oncol 3 : 399408, 2005

32. The British Pain Society : Intrathecal drug delivery for the management of pain and spasticity in adults; recommendations for best clinical practice. London : The British Pain Society, 2008. Available from : http ://www. britishpainsociety.org/book_ittd_main.pdf. Accessed September 23, 2014

33. Wilkes D : Programmable intrathecal pumps for the management of chronic pain : recommendations for improved efficiency. J Pain Res 7 : 571-577, 2014 